\title{
Management and Outcomes of Patients with Recurrent Intrahepatic Cholangiocarcinoma Following Previous Curative- Intent Surgical Resection
}

\author{
Gaya Spolverato, MD ${ }^{1}$, Yuhree Kim, MD, MPH ${ }^{1}$, Sorin Alexandrescu, MD ${ }^{2}$, Hugo P. Marques, $\mathrm{MD}^{3}$, \\ Jorge Lamelas, $\mathrm{MD}^{3}$, Luca Aldrighetti, $\mathrm{MD}^{4}$, T. Clark Gamblin, $\mathrm{MD}^{5}$, Shishir K. Maithel, $\mathrm{MD}^{6}$, Carlo Pulitano, \\ $\mathrm{MD}^{7}$, Todd W. Bauer, $\mathrm{MD}^{8}$, Feng Shen, $\mathrm{MD}^{9}$, George A. Poultsides, $\mathrm{MD}^{10}$, Thuy B. Tran, $\mathrm{MD}^{10}$, J. Wallis Marsh, \\ $\mathrm{MD}^{11}$, and Timothy M. Pawlik, MD, MPH, PhD ${ }^{1,12}$ \\ ${ }^{1}$ The Johns Hopkins University School of Medicine, Baltimore, MD; ${ }^{2}$ Fundeni Clinical Institute, Bucharest, Romania; \\ ${ }^{3}$ Curry Cabral Hospital, Lisbon, Portugal; ${ }^{4}$ Ospedale San Raffaele, Milan, Italy; ${ }^{5}$ Medical College of Wisconsin, \\ Milwaukee, WI; ${ }^{6}$ Emory University, Atlanta, GA; ${ }^{7}$ University of Sydney, Sydney, Australia; ${ }^{8}$ University of Virginia, \\ Charlottesville, VA; ${ }^{9}$ Eastern Hepatobiliary Surgery Hospital, Shanghai, China; ${ }^{10}$ Stanford University, Stanford, CA; \\ ${ }^{11}$ University of Pittsburgh Medical Center, Pittsburgh, PA; ${ }^{12}$ Division of Surgical Oncology, John L. Cameron Professor of \\ Alimentary Surgery, Department of Surgery, Johns Hopkins Hospital, Baltimore, MD
}

\begin{abstract}
Background. Management and outcomes of patients with recurrent intrahepatic cholangiocarcinoma (ICC) following curative-intent surgery are not well documented. We sought to characterize the treatment of patients with recurrent ICC and define therapy-specific outcomes.

Methods. Patients who underwent surgery for ICC from 1990 to 2013 were identified from an international database. Data on clinicopathological characteristics, operative details, recurrence, and recurrence-related management were recorded and analyzed.

Results. A total of 563 patients undergoing curative-intent hepatic resection for ICC who met the inclusion criteria were identified. With a median follow-up of 19 months, 400 $(71.0 \%)$ patients developed a recurrence. At initial surgery,
\end{abstract}

This work was presented at ASCO GI Symposium in San Francisco, CA, January 15-17, 2015 and at SSO Annual Cancer Symposium in Huston, TX, March 25-28, 2015.

Electronic supplementary material The online version of this article (doi:10.1245/s10434-015-4642-9) contains supplementary material, which is available to authorized users.

(C) Society of Surgical Oncology 2015

First Received: 10 February 2015;

Published Online: 10 June 2015

T. M. Pawlik, MD, MPH, PhD

e-mail: tpawlik1@jhmi.edu treatment was resection only $(98.8 \%)$ or resection + ablation (1.2\%). Overall 5-year survival was $23.6 \%$; $400(71.0 \%)$ patients recurred with a median disease-free survival of 11.2 months. First recurrence site was intrahepatic only $(59.8 \%)$, extrahepatic only (14.5\%), or intra- and extrahepatic $(25.7 \%)$. Overall, $210(52.5 \%)$ patients received best supportive care (BSC), whereas $190(47.5 \%)$ patients received treatment, such as systemic chemotherapy-only $(24.2 \%)$ or repeat liver-directed therapy \pm systemic chemotherapy $(75.8 \%)$. Repeat liver-directed therapy consisted of repeat hepatic resection \pm ablation $(28.5 \%)$, ablation alone $(18.7 \%)$, and intra-arterial therapy (IAT) (52.8\%). Among patients who recurred, median survival from the time of the recurrence was 11.1 months (BSC 8.0 months, systemic chemotherapy-only 16.8 months, liverdirected therapy 18.0 months). The median survival of patients undergoing resection of recurrent ICC was 26.7 months versus 9.6 months for patients who had IAT $(p<0.001)$.

Conclusions. Recurrence following resection of ICC was common, occurring in up to two-thirds of patients. When there is recurrence, prognosis is poor. Only $9 \%$ of patients underwent repeat liver resection after recurrence, which offered a modest survival benefit.

Intrahepatic cholangiocarcinoma (ICC) is an aggressive neoplasm arising from the epithelial cells of the intrahepatic bile ducts. ICC accounts for 10-15\% of all primary liver cancers and is the second most common primary liver tumor after hepatocellular carcinoma. ${ }^{1,2}$ The incidence of 
ICC has increased in the past 30 years from 0.32 per 100,000 to 0.85 per 100,000 worldwide and similarly from 0.13 per 100,000 to 0.67 per 100,000 in the United States. ${ }^{3,4}$ Mortality associated with ICC also has increased over time from 0.7 per $1,000,000$ in the 1970 s to 6.9 per $1,000,000$ in 2000, paralleling the increase in the incidence of ICC. ${ }^{4}$

Many patients with ICC present with advanced disease, characterized by large tumor size, vascular invasion, and multifocality. ${ }^{5}$ As such, only $20-40 \%$ of patients with ICC are considered potentially operable at the time of presentation and are offered surgical resection. ${ }^{6,7}$ When feasible, hepatic resection remains the best potential curative treatment for ICC. However, even after curative-intent surgery, the 5-year survival of patients undergoing liver resection ranges from only $30-35 \%$, with a median overall survival of approximately 28 months. ${ }^{5}$ The addition of adjuvant chemotherapy and/or radiotherapy with surgery has similarly failed to offer a durable survival benefit for the majority of patients; less than one-third of these patients survive beyond 5 years. ${ }^{5,8,9}$

Data for risk factors associated with recurrence, as well as data characterizing the treatment and outcome of patients with recurrent ICC are lacking. Specifically, few studies have evaluated the rates and patterns of recurrence after hepatic resection for ICC. Most of these studies did not report the management of recurrence, nor did these studies define the therapy-specific outcomes for recurrent disease. ${ }^{10-15}$ Furthermore, most of these studies suffered from a sample size and were based on single-institution experience. ${ }^{11,12,16}$

During the past decade, there has been increasing interest in the surgical management of recurrent hepatobiliary malignancies. Several studies have demonstrated the efficacy of repeat surgical resection or ablation for the treatment of colorectal liver metastasis and hepatocellular carcinoma; however, there is a paucity of data on the management of recurrent ICC. ${ }^{16-18}$ Given this, the objective of the current study was to characterize the treatment of patients with recurrent ICC and define therapy-specific outcomes. Specifically, using a large multi-institutional cohort of patients, we sought to determine the rate and the pattern of recurrent disease following curative-intent surgery for ICC, as well as define the therapy-specific outcome of patients treated for recurrent disease.

\section{MATERIALS AND METHODS}

\section{Patient Demographic and Clinical Data}

Patients undergoing curative-intent hepatic resection for ICC between 1990 and 2013 at 1 of 12 major hepatobiliary centers in the United States, Europe, Australia, and Asia (Johns Hopkins Hospital, Baltimore, MD; Medical College of Wisconsin, Milwaukee, WI; Stanford University, Stanford, CA; University of Virginia, Charlottesville, VA; Emory University, Atlanta, GA; University of Pittsburgh, Pittsburgh, PA; Fundeni Clinical Institute of Digestive Disease, Bucharest, Romania; Curry Cabral Hospital, Lisbon, Portugal; Hopitaux Universitaires De Geneve, Geneve, Switzerland; Ospedale San Raffaele, Milan, Italy; Royal Prince Alfred Hospital, University of Sydney, Sydney, Australia; Eastern Hepatobiliary Surgery Hospital, Shanghai, China) were identified. Only patients with histologically confirmed ICC were included in the study group. Patients who underwent a palliative operation, had metastatic disease, or experienced perioperative mortality within 30 days of surgery were excluded from analysis. Patients who underwent only ablation or intra-arterial therapy (IAT) as treatment of the primary tumor were also excluded. The Institutional Review Board of each institution approved the study.

Standard patient demographic and clinicopathologic data were collected, including age, sex, presence of cirrhosis, and tumor-specific characteristics. In particular, data were collected on primary tumor size, number, grade, lymph node status, and presence of vascular invasion (macroscopic and microscopic), perineural invasion, biliary invasion, and direct invasion of contiguous organs. Data on tumor stage were also collected according to 7th edition AJCC staging system. ${ }^{19}$ Resection margin status was recorded and classified as microscopically negative (R0), microscopically positive (R1), or macroscopically positive (R2). Data regarding treatment details of the primary tumor also were collected, including type of surgery and receipt of adjuvant chemotherapy and radiotherapy. Perioperative complications and mortality were considered within 90 days from the operation. ${ }^{20}$ Complications were categorized based on the Clavien-Dindo classification system, with minor complications defined as grade I or II and major complications as grade III or IV. ${ }^{21}$

Date of last follow-up, vital status, and recurrence-related information were collected on all patients. Recurrence was defined as the presence of a biopsy-proven tumor or an image that was highly suspicious of tumor recurrence. In general, similar to previous studies, patients had surveillance imaging every 3-4 months for the first 1-3 years and every 6 months from 3 to 5 years. ${ }^{22-24}$ Information regarding the location and number of lesions, as well as the disease-free interval from the date of initial operation to the development of recurrent disease was recorded. For the cohort of patients who developed repeat recurrences, data on the pattern and time interval between subsequent recurrences also were noted. Recurrences were classified as intrahepatic or extrahepatic, and the specific site of recurrence was recorded 
(lung, adrenal, bone, peritoneum, etc.) when available. Data on treatment of recurrent ICC also were collected and for the purpose of the study were classified as best supportive care (BSC), liver-directed therapy, and chemotherapy-only. Liver-directed therapy was considered as hepatic resection \pm ablation, ablation only, and IAT; selection of patients for liver-directed therapy was at the discretion of the treating surgeon.

\section{Statistical Analysis}

Discrete variables were described as medians with interquartile range (IQR) and categorical variables were described as totals and frequencies. Univariate comparisons were assessed using the chi-squared test or Fisher's exact test as appropriate. Univariate and multivariate logistic regression models were assessed to determine factors associated with "any site" recurrence and site-specific recurrence. Variables with univariate significance $(P<0.05)$ were entered into the multivariate model. Overall survival (OS) for the study population and recurrence-free survival (RFS) were estimated using the Kaplan-Meier method, and the differences in OS and RFS were assessed with the log-rank test. All analyses were performed with STATA version 12.0 (StataCorp, College Station, TX), and $P<0.05$ (two-tailed) was considered statistically significant.

\section{RESULTS}

\section{Clinicopathological and Treatment Characteristics}

A total of 563 patients undergoing curative-intent hepatic resection for ICC who met the inclusion criteria were identified. With a median follow-up of 19 months, $400(71.0 \%)$ patients developed a recurrence. Baseline characteristics of the population that recurred after curative-intent therapy for ICC are summarized in Supplementary Table 1. The median patient age was 59 years (IQR 49-69); the majority of patients were male $(n=213,53.4 \%)$ and non-Hispanic white ( $n=296,78.3 \%$ ). A minority of patients had cirrhosis of the background liver $(n=47,11.8 \%)$. The median size of the largest tumor was $7 \mathrm{~cm}$ (IQR $5.0-9.5 \mathrm{~cm}$ ). One third of tumors were solitary ( $n=137,34.9 \%)$, and the median tumor burden was 1 (IQR 1-2). At the time of surgery, the majority of patients underwent a hemihepatectomy $(n=175$, $44.1 \%)$. The remaining patients underwent either less than a hemihepatectomy $(n=126 ; 31.7 \%)$ or extended hemihepatectomy ( $n=96 ; 24.2 \%)$. Only $5(1.2 \%)$ patients underwent a concomitant liver ablation plus resection. Complete R0 resection was achieved in the majority of patients $(n=328,82.4 \%)$. On the final pathological evaluation, almost one-quarter of patients presented with vascular
TABLE 1 Patterns of recurrence following curative-intent surgery of intrahepatic cholangiocarcinoma

\begin{tabular}{ll}
\hline Characteristic & Recurrence $(n=400)$ \\
\hline Intrahepatic only & $239(59.8)$ \\
Extrahepatic only & $58(14.5)$ \\
Intrahepatic and extrahepatic & $103(25.7)$ \\
Site of extrahepatic recurrence & $n=161$ \\
Lung & $48(29.8)$ \\
Adrenal & $4(2.5)$ \\
Bone & $16(9.9)$ \\
Peritoneum & $34(21.1)$ \\
Lymph node & $38(23.6)$ \\
Other & $21(13.0)$ \\
\hline
\end{tabular}

invasion (microvascular invasion: $n=82,20.5 \%$; macrovascular invasion: $n=19,4.8 \%$; both: $n=33,8.2 \%$ ). Biliary invasion $(n=59,14.8 \%)$ and direct invasion of contiguous organs $(n=43,10.8 \%)$ were less common. The majority of patients had a mass-forming ICC $(n=280$, $80.4 \%)$ and a moderately differentiated $(n=241,61.6 \%)$ or poorly differentiated/undifferentiated tumor $(n=107$, $27.4 \%$ ). Less than one-quarter of patients was found to have lymph node metastasis ( $n=74,18.5 \%$ ). Among those patients who experienced recurrence and had information on AJCC stage, the majority was either stage I $(n=81,32.4 \%)$ or stage IVa $(n=86,34.4 \%)$. A total of $176(44.0 \%)$ patients experienced a postoperative complication. Among these patients, 106 (59.9\%) had a minor complication, whereas $71(40.1 \%)$ had a major complication. Half of patients received adjuvant chemotherapy $(n=201,50.2 \%)$, whereas only a small subset $(n=44,11.0 \%)$ received adjuvant radiotherapy.

\section{Patterns and Predictors of Recurrence}

Among the 400 patients who experienced a recurrence, $239(59.8 \%)$ developed only intrahepatic disease, whereas a minority $(n=58,14.5 \%)$ had only extrahepatic disease and $103(25.7 \%)$ had both intra- and extrahepatic disease. The most common site of extrahepatic recurrence was lung (29.8\%), followed by distant lymph nodes $(23.6 \%)$, and peritoneum (21.1\%; Table 1).

Median, 1-, 3-, and 5-year RFS was 11.2 months, 47.8, 22.4, and $17.4 \%$ respectively. The median RFS was independent from the pattern of recurrence (intrahepatic only: 7.4 months vs. extrahepatic only: 8.9 months, $P=$ 0.33). Among patients who experienced a recurrence, virtually all recurrences occurred within 5 years, with the highest risk being within the first 2 years of surgery (Fig. 1). Several patient and tumor-related factors were associated with "any site" recurrence, including presence 
of cirrhosis in the background liver, tumor size and number, vascular invasion, tumor grade, as well as lymph node status (Table 2). Specifically, patients who experienced a recurrence were more likely to have cirrhosis (hazard ratio [HR] 1.62, $95 \%$ confidence interval [CI] 1.17-2.25; $P=$ 0.004), multiple lesions (HR 1.43, $95 \%$ CI 1.15-1.78; $P=$ 0.001 ), and large tumors (HR 1.38, $95 \%$ CI 1.11-1.70; $P=$ 0.003 ). Evidence of microvascular invasion (HR 1.56, $95 \%$ CI 1.21-2.02; $P=0.001)$ and macrovascular invasion (HR 1.60, $95 \%$ CI 1.06-2.42; $\mathrm{P}=0.025$ ) were each associated with a higher risk of "any site" recurrence. In addition, the presence of lymph node metastasis (HR 1.46, $95 \%$ CI $1.08-1.97 ; P<0.001)$ and poor differentiation (HR 1.30, $95 \%$ CI 1.03-1.64; $P=0.024$ ) similarly increased the risk of recurrence. Patients with periductalinfiltrating tumor (HR 1.70, $95 \%$ CI 1.05-2.76; $P=0.032$ ) and those with mass-forming morphology \pm periductalinfiltrating aspects (HR 1.74, $95 \%$ CI 1.16-2.63; $P=$ 0.008 ) also were more likely to experience recurrence.

In examining the pattern of recurrence, several factors were associated with the specific patterns of recurrence (Table 2). For example, patients with cirrhosis were at increased risk of intrahepatic recurrence (HR 1.91, $95 \%$ CI 1.31-2.80; $P=0.001$ ). Similarly multiple lesions (HR $1.34,95 \%$ CI 1.01-1.78; $P=0.04$ ) or a large tumor (HR $1.53,95 \%$ CI $1.17-2.00 ; P=0.002$ ) were independently associated with higher risk of intrahepatic recurrence. In addition, patients with mass-forming tumor \pm periductalinfiltrating morphology were more likely to develop intrahepatic recurrence (HR 1.61, $95 \%$ CI 1.00-2.60; $P=$ 0.049). Extrahepatic recurrence was associated with several factors, including micro- and macrovascular invasion, R1 margin, perineural or biliary invasion, tumor grade, periductal-infiltrating morphology, as well as lymph node metastasis (all $P<0.05$; Table 2). After controlling for competing risk factors, only the presence of lymph node metastasis (HR 2.06, $95 \%$ CI 1.30-3.26; $P=0.002$ ) and periductal-infiltrating morphology (HR 2.48, $95 \%$ CI 1.13-5.45; $P=0.023$ ) remained associated with an increased risk of extrahepatic recurrence.

\section{Treatment of Recurrence and Long-term Outcomes}

Overall, $190(47.5 \%)$ patients received treatment for recurrent ICC, whereas $210(52.5 \%)$ received BSC. Among patients who received treatment for recurrent disease, therapy consisted of systemic chemotherapy only ( $n=$ $46,24.2 \%$ ) or repeat liver-directed therapy \pm systemic chemotherapy ( $n=144,75.8 \%)$. Repeat liver-directed therapy consisted of repeat hepatic resection \pm ablation $(n=41,28.5 \%)$, ablation alone $(n=27,18.7 \%)$, or IAT $(n=$ 76, $52.8 \%$; Supplementary Figure).
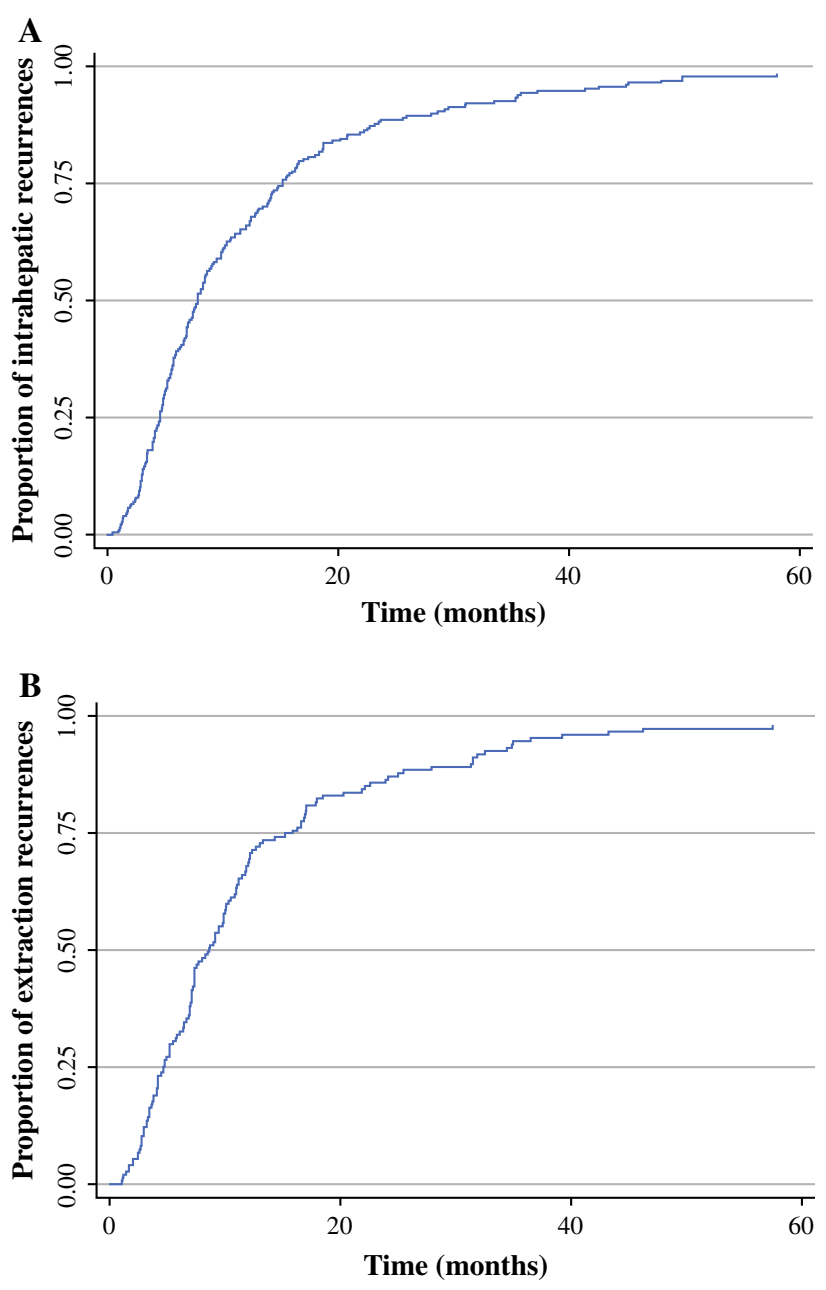

FIG. 1 Five-year overall risk of recurrence stratified by site of disease: intrahepatic (a), extrahepatic (b)

Older patients were more likely to undergo BSC (median age: 61.2 years, IQR 53.0-70.0) than liver-directed therapy (median age: 55.5 years, IQR 45.8-65.6) or chemotherapy only (median age: 57.3 years, IQR 49.0 69.9; $P=0.001$; Table 3). Patients who received repeat liver-directed therapy were more likely to have a less aggressive primary ICC, such as moderately differentiated, stage I-II tumor, and no lymph node metastasis (all $P<$ 0.05). Among patients with extrahepatic recurrence, the most common treatment was systemic chemotherapy; in contrast, patients with intrahepatic recurrence only were more likely to receive repeat liver-directed therapy $(P<$ 0.001). Interestingly, size and number of the recurrent lesions did not correlate with different therapeutic approaches $(P<0.05)$.

Among patients who recurred, median survival from the time of the recurrence was 11.1 months (BSC 8.0 months; systemic chemotherapy only 16.8 months; liver-directed therapy 18.0 months; $P<0.001$; Fig. 2). The median survival of patients undergoing resection of recurrent ICC 
TABLE 2 Univariable and multivariable cox proportional hazards models to predict (a) "any site" recurrence, (b) intrahepatic recurrence, and (c) extrahepatic recurrence

\begin{tabular}{|c|c|c|c|c|}
\hline & \multicolumn{2}{|l|}{ Univariate } & \multicolumn{2}{|l|}{ Multivariate } \\
\hline & $\mathrm{HR}(95 \% \mathrm{CI})$ & $P$ value & $\mathrm{HR}(95 \% \mathrm{CI})$ & $P$ value \\
\hline \multicolumn{5}{|l|}{ (a) "Any site" recurrence } \\
\hline Bilobar lesions & $1.35(1.09-1.67)$ & 0.006 & $1.23(0.98-1.55)$ & 0.075 \\
\hline Cirrhosis & $2.04(1.38-3.01)$ & 0.02 & $1.62(1.17-2.25)$ & 0.004 \\
\hline Multiple lesions & $1.60(1.29-1.97)$ & $<0.001$ & $1.43(1.15-1.78)$ & 0.001 \\
\hline Size $\geq 7 \mathrm{~cm}$ & $1.51(1.23-1.84)$ & $<0.001$ & $1.38(1.11-1.70)$ & 0.003 \\
\hline \multicolumn{5}{|l|}{ Vascular invasion } \\
\hline No vascular invasion & Ref & & Ref & \\
\hline Microvascular invasion & $1.75(1.36-2.25)$ & $<0.001$ & $1.56(1.21-2.02)$ & 0.001 \\
\hline Macrovascular invasion & $1.88(1.16-3.03)$ & 0.01 & $1.41(0.83-2.41)$ & 0.206 \\
\hline Both micro- and macrovascular invasion & $1.62(1.12-2.36)$ & 0.011 & $1.60(1.06-2.42)$ & 0.025 \\
\hline Direct invasion of contiguous organs & $1.42(1.03-1.97)$ & 0.033 & $1.10(0.74-1.63)$ & 0.646 \\
\hline \multicolumn{5}{|l|}{$\mathrm{N}$ stage } \\
\hline No & Ref & & Ref & \\
\hline N1 & $1.56(1.20-2.02)$ & 0.001 & $1.46(1.08-1.97)$ & 0.012 \\
\hline \multicolumn{5}{|l|}{ T stage } \\
\hline $\mathrm{T} 1-\mathrm{T} 2$ & Ref & & Ref & \\
\hline T3-T4 & $1.29(1.01-1.66)$ & 0.043 & $0.96(0.71-1.30)$ & 0.811 \\
\hline \multicolumn{5}{|l|}{ Histological grade } \\
\hline Well to moderately differentiated & Ref & & Ref & \\
\hline Poorly to undifferentiated & $1.40(1.11-1.75)$ & 0.004 & $1.30(1.03-1.64)$ & 0.024 \\
\hline \multicolumn{5}{|l|}{ Morphologic type } \\
\hline Mass-forming & Ref & & Ref & \\
\hline Papillary & $0.69(0.42-1.15)$ & 0.16 & $0.82(0.49-1.38)$ & 0.452 \\
\hline Periductal-infiltrating & $1.56(0.98-2.49)$ & 0.059 & $1.70(1.05-2.76)$ & 0.032 \\
\hline Mass-forming \pm periductal infiltrating & $1.59(1.10-2.28)$ & 0.013 & $1.74(1.16-2.63)$ & 0.008 \\
\hline Adjuvant chemotherapy & $1.03(0.83-1.26)$ & 0.809 & & \\
\hline Adjuvant radiotherapy & $1.00(0.72-1.38)$ & 0.983 & & \\
\hline \multicolumn{5}{|l|}{ (b) Intrahepatic recurrence } \\
\hline Cirrhosis & $1.77(1.22-2.57)$ & 0.003 & $1.91(1.31-2.80)$ & 0.001 \\
\hline Multiple lesions & $1.43(1.08-1.88)$ & 0.012 & $1.34(1.01-1.78)$ & 0.04 \\
\hline Size $\geq 7 \mathrm{~cm}$ & $1.55(1.19-2.01)$ & 0.001 & $1.53(1.17-2.00)$ & 0.002 \\
\hline \multicolumn{5}{|l|}{ Morphologic type } \\
\hline Mass-forming & Ref & & Ref & \\
\hline Papillary & $0.43(0.19-0.98)$ & 0.045 & $0.50(0.22-1.15)$ & 0.102 \\
\hline Periductal-infiltrating & $0.99(0.48-2.00)$ & 0.97 & $0.90(0.44-1.83)$ & 0.771 \\
\hline Mass-forming \pm periductal infiltrating & $1.57(0.98-2.53)$ & 0.061 & $1.61(1.00-2.60)$ & 0.049 \\
\hline Adjuvant chemotherapy & $0.94(0.72-1.23)$ & 0.643 & & \\
\hline Adjuvant radiotherapy & $1.42(0.90-2.26)$ & 0.133 & & \\
\hline \multicolumn{5}{|l|}{ (c) Extrahepatic recurrence } \\
\hline \multicolumn{5}{|l|}{ Vascular invasion } \\
\hline No vascular invasion & Ref & & Ref & \\
\hline Microvascular invasion & $1.80(1.22-2.65)$ & 0.003 & $1.51(0.99-2.30)$ & 0.057 \\
\hline Macrovascular invasion & $2.11(1.02-4.35)$ & 0.044 & $1.77(0.84-3.76)$ & 0.134 \\
\hline Both micro- and macrovascular invasion & $1.94(1.12-3.36)$ & 0.018 & $1.47(0.80-2.70)$ & 0.212 \\
\hline $\mathrm{R} 1$ margin & $1.56(1.05-2.33)$ & 0.028 & $1.19(0.78-1.81)$ & 0.429 \\
\hline Perineural invasion & $1.80(1.24-2.62)$ & 0.002 & $1.30(0.85-1.97)$ & 0.224 \\
\hline
\end{tabular}


TABLE 2 continued

\begin{tabular}{|c|c|c|c|c|}
\hline & \multicolumn{2}{|l|}{ Univariate } & \multicolumn{2}{|l|}{ Multivariate } \\
\hline & $\mathrm{HR}(95 \% \mathrm{CI})$ & $P$ value & $\mathrm{HR}(95 \% \mathrm{CI})$ & $P$ value \\
\hline Biliary invasion & $1.72(1.15-2.58)$ & 0.008 & $1.26(0.81-1.97)$ & 0.3 \\
\hline \multicolumn{5}{|l|}{$\mathrm{N}$ stage } \\
\hline No & Ref & & Ref & \\
\hline N1 & $1.95(1.31-2.89)$ & 0.001 & $2.06(1.30-3.26)$ & 0.002 \\
\hline \multicolumn{5}{|l|}{ Histological grade } \\
\hline Well to moderately differentiated & Ref & & Ref & \\
\hline Poorly to undifferentiated & $1.53(1.07-2.19)$ & 0.02 & $1.39(0.96-2.01)$ & 0.077 \\
\hline \multicolumn{5}{|l|}{ Morphologic type } \\
\hline Mass-forming & Ref & & Ref & \\
\hline Papillary & $1.48(0.74-2.95)$ & 0.262 & $1.47(0.72-3.00)$ & 0.294 \\
\hline Periductal-infiltrating & $2.46(1.24-4.90)$ & 0.01 & $2.48(1.13-5.45)$ & 0.023 \\
\hline Mass-forming \pm periductal infiltrating & $1.31(0.66-2.60)$ & 0.446 & $1.17(0.55-2.48)$ & 0.674 \\
\hline Adjuvant chemotherapy & $1.05(0.75-1.47)$ & 0.767 & & \\
\hline Adjuvant radiotherapy & $0.65(0.41-1.03)$ & 0.064 & & \\
\hline
\end{tabular}

was 26.1 months versus 25.5 months for patients who underwent ablation only and 9.6 months for patients who had IAT $(P=0.01)$. More than half of patients $(53.6 \%)$ who underwent a repeat resection \pm ablation experienced a second recurrence with a median time to second recurrence of 11.5 months.

\section{DISCUSSION}

Intrahepatic cholangiocarcinoma is a rare, life-threatening malignancy, which generally has a poor prognosis. In fact, data from the current paper found that recurrence following curative intent surgery for ICC was extremely high. After surgery, less than one in five patients was alive and free of disease 5 years following the date of surgery. Given the high incidence of recurrent disease, understanding risk factors associated with recurrence andperhaps more importantly - the pattern, as well as management, of recurrent ICC disease is critical. The current study is important, because-using a large, international, multi-institutional cohort of patients-we defined the incidence of recurrence to be $73.1 \%$ at 5 years, as well as characterized the overall pattern of ICC recurrence following curative intent surgery. More than half of patients developed recurrence within 1 year, and the median RFS was only 11.2 months. Of note, the most common pattern of recurrence was intrahepatic $(59.8 \%)$, whereas fewer patients had an extrahepatic component of recurrent disease $(40.2 \%)$. Although ICC most often recurred in the liver, only half of patients were suitable for treatment at the time of recurrence. Interestingly, among those patients who underwent repeat liver-directed therapy, outcomes were poor with more than half of patients experiencing a second recurrence within 12 months following repeat resection.

Despite the curative-intent of surgery, recurrence was common following surgical resection; only $17.4 \%$ of patients were recurrence-free at 5 years. In fact, with a median follow-up of 19 months, $71.0 \%$ of patients had developed either an intra- or extrahepatic recurrence. These data corroborate previous reports that have similarly noted the aggressive natural history of ICC. ${ }^{5,10-15}$ Although the most common recurrence site was the liver, recurrence outside of the liver also was common (Table 1). Interestingly, median and 5-year RFS were comparable among patients who presented with either intra- or extrahepatic disease as the first site of their recurrence. We noted that factors, such as cirrhosis of the background liver, as well as specific morphological features, such as periductal-infiltrating tumor or mass-forming tumor \pm periductalinfiltrating morphology, were independent predictors of "any site" recurrence. Certain tumor-level factors also were associated with specific patterns of recurrence. For example, multifocal ICC and large tumor size were independent predictors of intrahepatic recurrence, whereas the presence of lymph node metastasis substantially increased the risk of extrahepatic disease. Data from our group and others have emphasized the importance of assessing the lymph node basin to stage patients accurately., ${ }^{9,25-27}$ The finding that lymph node metastasis were strongly associated with risk of extrahepatic recurrence serves to emphasize the importance of assessing the regional nodal basins. While lymph node metastasis may indeed portend a 
TABLE 3 Patient and tumor factors and recurrence patterns stratified by treatment of recurrence among patients with curative-intent therapy for intrahepatic cholangiocarcinoma

\begin{tabular}{|c|c|c|c|c|}
\hline & Best supportive care $(n=210)$ & Liver-directed therapy $(n=144)$ & Chemotherapy-only $(n=46)$ & $P$ \\
\hline Age & $61.2(53.0-70.0)$ & $55.5(45.8-65.5)$ & $57.3(49.0-69.9)$ & 0.001 \\
\hline $\operatorname{Sex}(n=399)$ & & & & 0.005 \\
\hline Male & $108(51.7)$ & $89(61.8)$ & $16(34.8)$ & \\
\hline Female & $101(48.3)$ & $55(38.2)$ & $30(65.2)$ & \\
\hline Cirrhosis & $14(6.7)$ & $30(20.8)$ & $3(6.5)$ & $<0.001$ \\
\hline Nodal status & & & & $<0.001$ \\
\hline Positive & $47(22.4)$ & $19(13.2)$ & $8(17.4)$ & \\
\hline Negative & $63(30.0)$ & $87(60.4)$ & $25(54.3)$ & \\
\hline Not harvested & $100(47.6)$ & $38(26.4)$ & $13(28.3)$ & \\
\hline AJCC T stage $(n=382)$ & & & & 0.507 \\
\hline $\mathrm{T} 1$ & $69(34.3)$ & $59(43.4)$ & $22(48.9)$ & \\
\hline $\mathrm{T} 2$ & $85(42.3)$ & $53(39.0)$ & $15(33.3)$ & \\
\hline T3 & $31(15.4)$ & $15(11.0)$ & $5(11.1)$ & \\
\hline T4 & $16(8.0)$ & $9(6.6)$ & $3(6.7)$ & \\
\hline $\operatorname{AJCC}(n=250)$ & & & & 0.002 \\
\hline I & $25(22.5)$ & $43(40.6)$ & $13(39.4)$ & \\
\hline II & $29(26.1)$ & $34(32.1)$ & $8(24.2)$ & \\
\hline III & $3(2.7)$ & $6(5.7)$ & $3(9.1)$ & \\
\hline IVa & $54(48.6)$ & $23(21.7)$ & $9(27.3)$ & \\
\hline Tumor grade $(n=391)$ & & & & 0.149 \\
\hline Well & $28(13.5)$ & $10(7.1)$ & $5(11.9)$ & \\
\hline Moderate & $117(56.2)$ & $96(68.1)$ & $28(66.7)$ & \\
\hline Poor or undifferentiated & $63(30.3)$ & $35(24.8)$ & $9(21.4)$ & \\
\hline R0 margin & $163(78.4)$ & $126(87.5)$ & $39(84.8)$ & 0.272 \\
\hline Recurrence site & & & & $<0.001$ \\
\hline Intrahepatic only & $109(51.9)$ & $117(81.2)$ & $13(28.3)$ & \\
\hline Extrahepatic only & $39(18.6)$ & $3(2.1)$ & $16(34.8)$ & \\
\hline Intrahepatic + extrahepatic & $62(29.5)$ & $24(16.7)$ & $17(37.0)$ & \\
\hline Size of recurrent tumor & NA & $4(2.4-8.1)$ & $3.0(2.4-3.7)$ & 0.450 \\
\hline Number of recurrent lesions & $2(2-2)$ & $1(1-2)$ & $1(1-2)$ & 0.950 \\
\hline
\end{tabular}

higher risk of extrahepatic recurrence and indicate the need for adjuvant therapy, postoperative therapy unfortunately remains largely ineffective. For example, in the current study, use of adjuvant treatment, including chemotherapy and/or radiotherapy, was not protective from "any site" recurrence or site-specific recurrence (Table 2). These data support previous reports noting the marginal beneficial effect of adjuvant therapy on the long-term oncological outcome of patients with resected ICC. ${ }^{8,9}$

While repeat hepatectomy has been advocated as the treatment of choice for recurrent colorectal liver metastasis and some patients with recurrent hepatocellular carcinoma, few studies have specifically explored different management options for patients with recurrent ICC. $^{16,17}$ Interestingly, only half of patients with recurrent disease were considered suitable for treatment, such as systemic chemotherapy or liver-directed therapy, while the remaining patients were managed with BSC only (Supplementary Figure). Perhaps not surprisingly, older patients and those with advanced disease were more likely to receive BSC, whereas patients who had a history of a less aggressive primary tumor (e.g., earlier stage, no nodal metastasis, lower tumor stage) and intrahepatic only recurrence more often underwent repeat liver-directed therapy. Repeat liverdirected therapy most often consisted of IAT, followed by resection \pm ablation and ablation only (Supplementary Figure). IAT has been previously described as a potentially effective therapy for patients with primary, unresectable ICC. Hyder et al. reported 198 patients with advanced ICC treated with IAT and reported a median overall survival of 13.2 months. ${ }^{28}$ In the current study, the use of IAT for recurrent ICC was associated with a comparable-albeit 


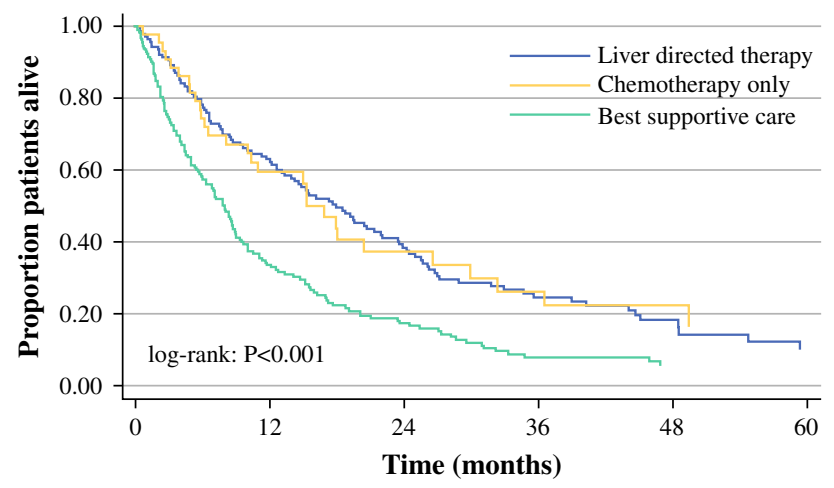

FIG. 2 Five-year overall survival stratified by type of treatment of recurrent disease

somewhat shorter-survival of just under 10 months. In contrast, the survival of patients undergoing resection or ablation was longer at 25-26 months. While this was considerably longer than IAT or BSC, it is important to note that the benefit of resection was not particularly durable. Collectively, the data strongly suggest that while repeat resection may be considered for a select subgroup of patients with recurrent intrahepatic ICC, the benefits of surgery are modest.

The current study had several limitations. Due to the retrospective nature, there may be selection/detection biases in our ascertainment of the pattern of recurrence. Although all the participating surgical departments queried institution-wide databases for information on recurrence and treatment, due to the surgical nature of the collaboration and of the dataset, recurrence may be underreported. However, these data should be missing at random and not affect the underlying findings of the current study. In addition, because this study was a multi-institutional collaboration among 12 academic institutions, operative and follow-up treatment protocols were not rigorously standardized. However, using a multi-institutional study cohort also was a major strength, because it conferred a larger sample size and more generalizable results. Another limitation of the study was that we included patients who underwent surgery for ICC in a large time period, between 1990 and 2013. This could have led to differences in diagnostic, therapeutical, and follow-up protocols in the same institution and between the different institutions.

\section{CONCLUSIONS}

Recurrence following curative-intent resection of ICC is common, occurring in up to two-thirds of patients within approximately 12 months of surgery. Intrahepatic recurrence was the most common pattern of initial recurrence, although extrahepatic recurrence also was frequent. The most common treatment of recurrent disease was BSC, while IAT was the most common liver-directed treatment. Only $9 \%$ of all patients underwent repeated liver resection when there was a recurrence. Resection was associated with only a modest survival benefit, because most patients developed a second recurrence within 12 months.

ACKNOWLEDGMENTS The authors thank Donielle Neal, Susana Rodrigues, John Miura, Francesca Ratti, Timothy Newhook, Malcolm H. Squires, and Stéphanie Meyer.

CONFLICT OF INTEREST None.

\section{REFERENCES}

1. Aljiffry M, Abdulelah A, Walsh M, Peltekian K, Alwayn I, Molinari M. Evidence-based approach to cholangiocarcinoma: a systematic review of the current literature. J Am Coll Surg. 2009;208(1):134-47.

2. Khan SA, Toledano MB, Taylor-Robinson SD. Epidemiology, risk factors, and pathogenesis of cholangiocarcinoma. $H P B$. 2008;10(2):77-82.

3. Shaib YH, Davila JA, McGlynn K, El-Serag HB. Rising incidence of intrahepatic cholangiocarcinoma in the United States: a true increase? J Hepatol. 2004;40(3):472-7.

4. Patel T. Increasing incidence and mortality of primary intrahepatic cholangiocarcinoma in the United States. Hepatology. 2001; 33(6):1353-7.

5. Mavros MN, Economopoulos KP, Alexiou VG, Pawlik TM. Treatment and prognosis for patients with intrahepatic cholangiocarcinoma: systematic review and meta-analysis. JAMA Surg. 2014. doi:10.1001/jamasurg.2013.5137.

6. Weber SM, Jarnagin WR, Klimstra D, DeMatteo RP, Fong Y, Blumgart LH. Intrahepatic cholangiocarcinoma: resectability, recurrence pattern, and outcomes. J Am Coll Surg. 2001;193(4): 384-91.

7. Amini N, Ejaz A, Spolverato G, Kim Y, Herman JM, Pawlik TM. Temporal trends in liver-directed therapy of patients with intrahepatic cholangiocarcinoma in the United States: a populationbased analysis. J Surg Oncol. 2014;110(2):163-70. doi:10.1002/ jso.23605.

8. Farges O, Fuks D, Boleslawski E, et al. Influence of surgical margins on outcome in patients with intrahepatic cholangiocarcinoma: a multicenter study by the AFC-IHCC-2009 study group. Ann Surg. 2011;254(5):824-9; discussion 830.

9. de Jong MC, Nathan H, Sotiropoulos GC, et al. Intrahepatic cholangiocarcinoma: an international multi-institutional analysis of prognostic factors and lymph node assessment. J Clin Oncol. $2011 ; 29(23): 3140-5$.

10. Nuzzo G, Giuliante F, Ardito F, et al. Intrahepatic cholangiocarcinoma: prognostic factors after liver resection. Updates Surg. 2010;62(1):11-9.

11. Chen LP, Li C, Wang C, Wen TF, Yan LN, Li B. Predictive factors of recurrence for patients with intrahepatic cholangiocarcinoma after hepatectomy. Hepato-gastroenterol. 2012;59(118):1765-8.

12. Hanazaki K, Kajikawa S, Shimozawa N, et al. Prognostic factors of intrahepatic cholangiocarcinoma after hepatic resection: univariate and multivariate analysis. Hepato-gastroenterol. 2002;49(44):3116.

13. Endo I, Gonen M, Yopp AC, et al. Intrahepatic cholangiocarcinoma: rising frequency, improved survival, and determinants of outcome after resection. Ann Surg. 2008;248(1):84-96. 
14. Miwa S, Miyagawa S, Kobayashi A, et al. Predictive factors for intrahepatic cholangiocarcinoma recurrence in the liver following surgery. J Gastroenterol. 2006;41(9):893-900.

15. Hyder O, Hatzaras I, Sotiropoulos GC, et al. Recurrence after operative management of intrahepatic cholangiocarcinoma. Surgery. 2013;153(6):811-8.

16. Kneuertz PJ, Cosgrove DP, Cameron AM, et al. Multidisciplinary management of recurrent hepatocellular carcinoma following liver transplantation. J Gastrointest Surg. 2012;16(4):874-81.

17. de Jong MC, Mayo SC, Pulitano C, et al. Repeat curative intent liver surgery is safe and effective for recurrent colorectal liver metastasis: results from an international multi-institutional analysis. J Gastrointest Surg. 2009;13(12):2141-51.

18. Maeda T, Hashimoto K, Ishida T, et al. Repeat hepatectomy for intrahepatic recurrence of cholangiolocellular carcinoma. Fukuoka igaku zasshi = Hukuoka acta medica. 2013;104(12): 564-8.

19. Edge SB BD, Compton CC, Fritz AG, Greene FL, Trotti A. AJCC cancer staging manual (7th edn). 2010.

20. Mayo SC, Shore AD, Nathan H, et al. Refining the definition of perioperative mortality following hepatectomy using death within 90 days as the standard criterion. HPB. 2011;13(7):473-82.

21. Dindo D, Demartines N, Clavien PA. Classification of surgical complications: a new proposal with evaluation in a cohort of 6336 patients and results of a survey. Ann Surg. 2004; 240(2):205-13.

22. Arnaoutakis DJ, Mavros MN, Shen F, et al. Recurrence patterns and prognostic factors in patients with hepatocellular carcinoma in noncirrhotic liver: a multi-institutional analysis. Ann Surg Oncol. 2014;21(1):147-54.

23. Spolverato G, Ejaz A, Kim Y, et al. Rates and patterns of recurrence after curative intent resection for gastric cancer: a United States multi-institutional analysis. $J$ Am Coll Surg. 2014;219(4):664-75. doi:10.1016/j.jamcollsurg.2014.03.062.

24. de Jong MC, Pulitano C, Ribero D, et al. Rates and patterns of recurrence following curative intent surgery for colorectal liver metastasis: an international multi-institutional analysis of 1669 patients. Ann Surg. 2009;250(3):440-8.

25. Amini N, Ejaz A, Spolverato G, Maithel SK, Kim Y, Pawlik TM. Management of lymph nodes during resection of hepatocellular carcinoma and intrahepatic cholangiocarcinoma: a systematic review. J Gastrointest Surg. 2014;18(12):2136-48.

26. Adachi T, Eguchi S, Beppu T, et al. Prognostic impact of preoperative lymph node enlargement in intrahepatic cholangiocarcinoma: a multi-institutional study by the Kyushu Study Group of Liver Surgery. Ann Surg Oncol. 2015;22:2269-78. doi:10.1245/s10434014-4239-8.

27. Guglielmi A, Ruzzenente A, Campagnaro T, et al. Patterns and prognostic significance of lymph node dissection for surgical treatment of perihilar and intrahepatic cholangiocarcinoma. $J$ Gastrointest Surg. 2013;17(11):1917-28.

28. Hyder O, Marsh JW, Salem R, et al. Intra-arterial therapy for advanced intrahepatic cholangiocarcinoma: a multi-institutional analysis. Ann Surg Oncol. 2013;20(12):3779-86. 